\title{
Green Tea Catechins -Pharmacokinetic Properties and Health Beneficial Effects
}

Jun-Ichiro Sonoda*, Keiko Narumi, Kawachi Akio, Tomishige Erisa,Motoya Toshiro

First Department of Clinical Pharmacy, School of Pharmaceutical Sciences, Kyushu University of Health \& Welfare, Nobeoka, Miyazaki, Japan

\begin{abstract}
In this review, we provide information on thepharmacokinetic properties of green tea catechins and their beneficial health effects. The major catechins in green tea are (-)-epicatechin (EC), its hydroxyl derivative (-)-epigallocatechin (EGC), and their gallic acid esters, (-)-epicatechin-3-gallate (ECg) and (-)-epigallocatechin-3-gallate (EGCg). We developed an analytical method for determination of the presence of green tea catechins in human serum using ion-pair HPLC with electrochemical detection to estimate the pharmacokinetic parameters of target catechins. The $\mathrm{C}_{\text {max }}$ values indicated that catechin absorption was relatively low. One of the gallatedcatechins, EGCg, had a longer half-life than the non-gallatedcatechins. Green tea catechins, in particular, have attracted attention as cancer preventive agents in terms of their low toxicity and being readily available to the general population. Several epidemiological studies revealed that green tea consumption reduces cancer incidence. Numerous in vitro cell culture studies have shown that EGCg, which is defined as a major green tea catechin contributing to green tea's anticancer effects, inhibits cell growth concomitant with induction of apoptosis. We have previously found that the cell death-inhibiting gene, $B C l-x L$, was decreased by EGCg. These results support the hypothesis that EGCg regulates cytoplasmic NF-KB and subsequently induction of apoptosis. Green tea consumption may also play a role in preventing other lifestyle diseases, such as cardiovascular diseases and stroke, due to its hypocholesterolemic and hypotensive activities. In conclusion, habitual green tea drinking may promote human health by preventing lifestyle-related diseases.
\end{abstract}

\section{Introduction}

The tea plant (Camellia sinensis), a native of southern China, has been known for more than two thousand years in Chinese botany and medicine.Lu Yu, who lived from 733 to 804 , is respected as the Sage of Tea for his contributions to Chinese tea culture. He is best known for his seminal book, Ch'aChing, or The Classic of Tea, the first definitive work on the cultivation, preparation, and drinking of tea.Eisai (1141-1215) was a Japanese Zen Buddhist monk who, after studying in China,brought back new tea seeds and introduced the tea ceremony. He also wrote the first book about the health benefits of drinking green tea, 'Kissa- Yohjoh-Ki' (Maintaining Health by Drinking Tea). Sen Rikyu (1522-1591) established the foundation of Chanoyu (The Japanese style of tea ceremony) which has been said to offer a comprehensive model for life. Currently in Japan, green tea is regarded as a healthy beverage by the public. The term 'green tea' refers to the product manufactured from fresh tea leaves by careful steaming or roasting to avoid oxidation of the polyphenolic components known as catechins. The major catechins in green tea are (-)-epicatechin (EC), its hydroxyl derivative(-)-epigallocatechin (EGC), and their respective gallic acid esters, (-)-epicatechin-3-gallate (ECg) and (-)-epigallocatechin-3-gallate (EGCg) (Figure 1). Among green tea catechins, EGCg is abundant in green tea leaves, and has beenshown to exhibit strong health-promoting activity[1,2], according to structure activity relationship assessment on EGCG, two close parallel aromatic rings and a third aromatic ring vertical to the two parallel rings may play a key role in the pharmacophore activity. This activity may be associated with the number of -OH groups in the catechin [3].

An understanding of the pharmacokinetic properties of green tea catechins is vital for promoting the human health benefits of its consumption. To assess the pharmacokinetics of green tea catechins after ingestion, we developed a new analytical method for detecting green tea catechins in serum. Pharmacokinetic study using this method showed individual variation of catechin serum concentration after ingestion of same amount of green tea catechins [4]. This observation indicated the existence of unknown factors affecting the pharmacokinetics of green tea catechins.

Numerous studies have shown that catechinshave anti-cancer, hypotensive, and hypocholesterolemic activities [5-10]. Consistently, inverse relationships between green tea consumption and the reduction of cancer incidence, the risk of developing hypertension, and cholesterol level have also been demonstrated in epidemiological studies.

Previously, the in vitro anti-cancer effects of a green tea extract on Adult T-cell Leukemia (ATL), which is an endemic disease caused by a latent infection of human retrovirus HTLV-1, have been demonstrated [11]. Furthermore, to investigate an intervention study, the in vivo effects of drinking green tea on HTLV-1 provirus load in asymptomatic HTLV-1 carriers have been conducted [12]. By integrating the results of these studies, we proposed a possible mechanism of the anti-cancer effect of EGCg was proposed in this review.

Habitual green tea drinking has been shown to reduce other lifestyle-related diseases. We review experimental and epidemiological evidence to demonstrate that green tea consumption is good for human health.

*Corresponding author: Sonoda JI, First Department of Clinical Pharmacy, School of Pharmaceutical Sciences, Kyushu University of Health \& Welfare Nobeoka, Miyazaki, 882-8508, Japan, Tel: +81982235529; Fax: +81982235536; E-mail: son@phoenix.ac.jp

Received November 15, 2014; Accepted January 27, 2015; Published February 02, 2015

Citation: Sonoda J, Narumi K, Akio K, Erisa T, Toshiro M (2015) Green Tea Catechins -Pharmacokinetic Properties and Health Beneficial Effects. Pharm Anal Acta 6: 333. doi:10.4172/2153-2435.1000333

Copyright: $\odot 2015$ Sonoda J, et al. This is an open-access article distributed under the terms of the Creative Commons Attribution License, which permits unrestricted use, distribution, and reproduction in any medium, provided the original author and source are credited. 
<smiles>Oc1cc(O)c2c(c1)O[C@H](c1ccc(O)c(O)c1)C[C@H]2O</smiles>

epicatechin (EC)<smiles>Oc1cc(O)c2c(c1)O[C@H](c1cc(O)c(O)c(O)c1)[C@H](O)C2</smiles>

epigallocatechin (EGC)<smiles>O=C(O[C@H]1Cc2c(O)cc(O)cc2O[C@H]1c1ccc(O)c(O)c1)c1cc(O)c(O)c(O)c1</smiles>

epicatechin gallate (ECG)<smiles>O=C(O[C@H]1Cc2c(O)cc(O)cc2O[C@H]1c1cc(O)c(O)c(O)c1)c1cc(O)c(O)c(O)c1</smiles>

epigallocatechin gallate (EGCG)
Figure 1: Structure of the four main catechins in green tea.

\section{Method of analysis for green tea catechins}

Although one regular cup of green tea contains several tens of milligrams of catechins, the serum concentration of catechins after ingestion reaches a maximum between several tens and several hundreds of $\mathrm{ng} / \mathrm{mL}[13]$. In order to assess the pharmacokinetics and beneficial health effects of catechins, a sensitive, reproducible, and straight-forward method is needed to quantify serum catechin concentration.

Generally, tea catechins are analyzed using high-performance liquid chromatography (HPLC) with ultraviolet (UV), chemiluminescence (CL), or electrochemical detection (ECD) also commonly used are capillary electrophoresis (CE) and gas chromatography with mass spectrometry (GC-MS)[14-18]. These methods have been used for determining catechin concentrations in tea, rat plasma, human plasma, and urine. Although GC-MS can be used for the determination of catechin concentration with high sensitivity, the required sample pretreatment is too demanding for routine analysis. HPLC is easier to use and more widely available. Previous work has shown that HPLCECD is more sensitive for the determination of catechin concentrations than HPLC-UV. Moreover, ECD is well suited to the detection of polyphenolic compounds like catechins because these analytes can be selectively oxidized by the appropriate electrical potential and flow current, the magnitude of this current is associated with the analyte concentration. Therefore, HPLC-ECD is an excellent candidate technique for the selective and highly sensitive detection of catechins in serum.

After ingestion of green tea, a large portion of catechins are present as glucuronate or sulfate conjugates in the serum.For the quantification of total catechins in serum, samples were treated with $\beta$-glucuronidase and sulfatase for hydrolysisto the free form of catechins. It has been reported that type $\mathrm{H}-2 \beta$-glucuronidase, which also contains sulfatase activity, effectively hydrolyzesconjugates in plasma samples but concomitantly cleavesthe ester bonds of EGCg and ECg. Therefore,these enzymes convertgallatedcatechins (EGCg and ECg) to their non-gallated forms (EGC and EC) $[5,19]$. Total catechins are expressed as EGCg + EGC and ECg + EC, which unfortunately makes it difficultto determine the exact concentrations of each individual catechin,due tosome EGC and EC beingderived from EGCg and ECg via treatment with the enzyme. Therefore, gallic acid (GA), derived from the gallatedcatechins, must be measured simultaneously to elucidate whether or not cleavage has occurred.

We developed a new simultaneous detection system of green tea catechins and GA in human serum using ion-pair HPLC withan ECD detection system. All the analytes (4 catechins and GA, and Ethyl gallate asan internal standard) were separated and obtained as single peaks, and the accuracy, reproducibility, and sensitivity of this method were sufficient to measure the target catechins and GA in serum [4].

\section{Pharmacokinetic properties of green tea catechins}

A pharmacokinetic study with 8 healthy volunteers was conducted using our method, and changes in the levels of the four catechinsand GA were monitored over time (Figure 2). No GA was detected in any of the samples. Other samples spiked with a known amount of standard catechins in phosphate buffer saline and treated with the deconjugation enzymes yielded a small amount of GA. The enzymatic decomposition of the gallatedcatechins may have been prevented by an unknown component in the serum. It has been reported that gallatedcatechins, specifically EGCg, preferentially bind with albumin in blood and can avoid metabolic conjugation [20,21]. The green tea extract tablets takenby subjectscontained $16.7 \mathrm{mg}$ of EC, $44.9 \mathrm{mg}$ of EGC, $11.1 \mathrm{mg}$ of ECg, and $42.9 \mathrm{mg}$ of EGCg, and mean $\mathrm{C}_{\max }(\mathrm{n}=8)$ reached $34.7,60.6,20.9$, and $42.8 \mathrm{ng} / \mathrm{mL}$, respectively. These $\mathrm{C}_{\max }$ values indicated that the amount of catechin absorption was relatively low. Gallatedcatechins such as EGCg and ECg had longer half-lives than the non-gallatedcatechins, EGC and EC, probably due to the majority of gallatedcatechins preferentially bindingto serum proteins and existing in the non-conjugated free form, delaying excretion. Moreover, efflux transporters, such as multidrug resistance-associated protein 2 (MRP2), a real so likely factors affecting half-life times; catechin metabolites are substrate of MRPs [22]. Li et al. demonstrated that non-gallatedcatechins were preferentially effluxed during their absorption across the small intestines using MRPs expressed in a Caco2 monolayer model, consistent with our observation [23]. Catechins may also be substrates of another transporter, p-glycoprotein (P-gp) [24]. Functional polymorphisms of P-gp have been shown both in human studies and in in vitro studies using cells stably transfected with various mutants of human P-gp [25]. Individual variation in $\mathrm{C}_{\max }$ and AUC of catechins after ingestion of green tea extract was observed in our pharmacokinetic study, which may be associated with the variation of P-gp activity among the subjects [26]. Recently, Misaka et al. reported a significant catechin-drug interaction; green tea ingestion greatly reduced plasma concentration of $\beta$-blocker nadolol mediated by organic anion-transporter OATP1A2 [27]. The catechindrug interaction competitive antagonism or direct inhibition for transporters should be kept in mind in the clinical field.

Fewer studies have reported the interaction between catechins and efflux transporters, possibly due to the instability of polyphenolic structures in cell culture medium or saline buffers caused by antioxidant level, $\mathrm{pH}$ of culture condition, concentration of proteins or the presence of metal ions [28]. To improve the stability of catechins in saline buffers, addition of a small amount of ascorbic acid (around 100 $\mu \mathrm{M})$ as an antioxidant is recommended [22,24].

\section{Anti-cancer effects of green tea drinking}

\section{Human studies}

General cancer: Imai et al. showed an association between green 


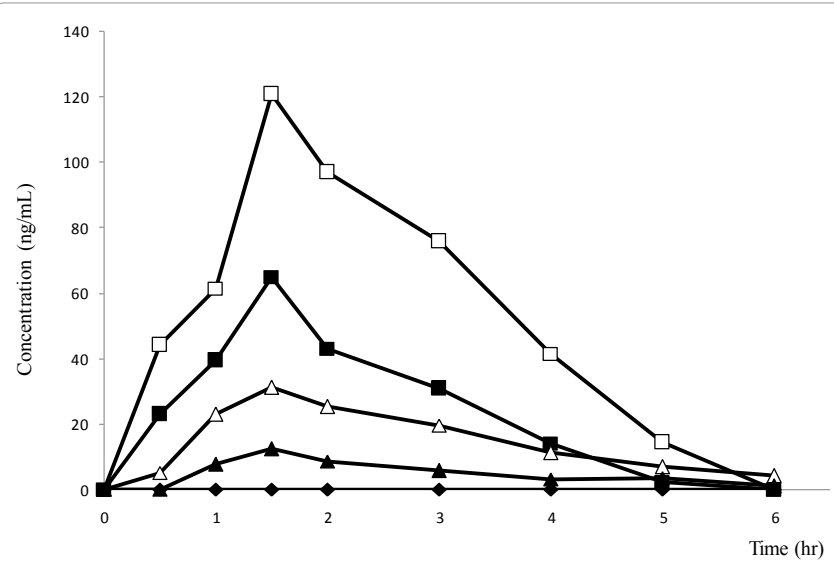

Figure 2: Mean serum concentration of the 4 target catechins versus time plots after ingestion of green tea tablets. Eight healthy volunteers ingested 3 tablets of decaffeinated green tea extract containing 4 major catechins. The amount of catechins in 3 tablets was equivalent to one regular size cup of green tea (EGC: $44.9 \mathrm{mg}$, EC: $16.7 \mathrm{mg}$, ECG: 11.1, EGCG: 42.9). Catechins:

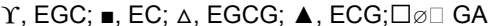

tea consumption and incidence of cancer in a prospective cohort study of a Japanese population with 8,552 individuals over the age of 40 residing in a town in Saitama Prefecture surveyed regarding their lifestyle habits, including their daily consumption of green tea [29]. An association between green tea consumption and cancer incidence was uncovered, with an increase in green tea consumption significantly decreasing the incidence of cancer among females. Although not statistically significant, males also showed a reduction in the relative risk of cancer with increased consumption of green tea, this was found among both smokers and nonsmokers. In a comparison of age-specific cancer incidence rates among females, an obvious reduction in agerelated increases in cancer incidence with increased green tea consumption was observed, especially for individuals in their $60 \mathrm{~s}$ and 70 s.

Prostate cancer: The association between green tea consumption and prostate cancer risk has been shown by Kurahasi et al. and the Japan Public Health Center-based Prospective Study Group. A total of 49,920 men were prospectively followed up (from 1990 to 2004); 404 men were newly diagnosed with prostate cancer, and of these, 114 were advanced cases, 271 were localized, and 19 were at an undetermined stage. Green tea consumption was associated with a dose-dependent decrease in the risk of developing advanced prostate cancer. The multivariate relative risk was 0.52 ( $95 \%$ confidence interval: $0.28,0.96$ ) for men drinking 5 or more cups/d compared with less than $1 \mathrm{cup} / \mathrm{d}\left(\mathrm{P}_{\text {trend }}=0.01\right)$. Thus, green tea consumption may be associated with a decreased risk of advanced prostate cancer[30]. Consistent results were shown in a oneyear proof-of-principle study of 60 volunteers with high-grade prostate intra-epithelial neoplasia (HG-PIN) [31]. Compared to the placebo group, the control group receiving $600 \mathrm{mg} / \mathrm{d}$ of green tea extract had reduced development of prostate cancer from HG-PIN. Notably, no significant side effects or adverse effects were found in the 1-year study period. These results indicate that long term ingestion of green tea extract is safe and effective for treating premalignant lesions before development of prostate cancer.

Breast cancer: Zhang et al. conducted a case-controlled study in southeast China to identify the protective effects of green tea consumption against breast cancer; 1009 women newly diagnosed with breast cancer were recruited to the study and an equal number of healthy women were selected as the control group. The dose-response relationships observed for the duration of green tea consumption and the number of cups consumed per day was similar. The author concluded that the regular consumption of green tea is associated with a reduced risk of breast cancer [32]. On the other hand, Suzuki et al. reported in a pooled analysis of two prospective studies with 35,000 Japanese women that there was no association between consumption of green tea and risk of breast cancer [33]. Because catechin absorption from the intestines to the bloodstream is relatively low [4], a high dose of about 10 cups/d in addition to along duration of green tea consumption maybe necessary to achieve an inverse association between green tea consumption and cancer incidence. Although inconsistent results have been observed in a case-controlled study and a prospective study, $\mathrm{Li}$ et al. showed that there was a statistically significant $19 \%$ reduction of breast cancer incidence among woman with high green tea intake in the most recent meta-analysis of two cohort studies and five casecontrolled studies [34-41].

Colorectal cancer: Yuan et al. have jointly conducted the Shanghai Cohort Study involving 18,244 men to investigate the association between urinary levels of EGC and its methyl metabolite (Me-EGC) as validated biomarkers of specific tea polyphenols and the risk of colorectal cancer. After a 16 year follow-up period, 162 subjects developed colorectal cancer. Among subjects with at least 5 years of follow-up, higher levels of Me-EGC were associated with lower risk of colon cancer. Compared with undetectable Me-EGC, odds ratios (95\% confidence interval) for colon cancer in the lowest, intermediate and highest tertiles of detectable Me-EGC were $0.49(0.25-0.96), 0.32$ $(0.16-0.67)$ and $0.41(0.20-0.84)$, respectively $\left(\mathrm{P}_{\text {trend }}=0.006\right)$; polyphenol biomarkers were not associated with rectal cancer risk. This study strongly supports long term green tea consumption for prevention of colon cancer in humans [42].

Lung cancer: A case-controlled study conducted in Okinawa, in the south of Japan, showed that daily tea consumption significantly decreased the risk of lung cancer, especially for squamous cell carcinoma [43]. Contrarily, Li et al. demonstrated in a populationbased cohort study in Miyagi, in northeastern Japan, that there was no association between green tea consumption and the risk of lung cancer [44]. To address these conflicting views, Wang et al. performed arecent meta-analysis of 38 lung cancer studies (26 case-controlled studies and 12 cohort studies) with 59,041 cases and 396,664 controls. The results showed that overall tea consumption was significantly associated with a decreased risk of lung cancer (RR, 0.78; 95\%CI, 0.70-0.87) [45].

Leukemia cancer: Based on epidemiological evidence and our preliminary experimental study [11], authors attempted to apply green tea for Adult T-cell Leukemia (ATL). The causative agent of ATL is the human retrovirus, HTLV-1. HTLV-1-infected individuals are prevalent world-wide but clustered the endemic area including southwestern Japan. Therefore, we investigated green tea as a chemo preventive agent in ATL development. We conducted an intervention study to investigate the in vivo effect of green tea on HTLV-1 provirus load in peripheral blood lymphocytes (PBLs) of HTLV-1 carriers. The subjects enrolled in this study were asymptomatic HTLV-1 carriers. They were randomly assigned to two groups. GT (-): no intake of any green tea capsules $(\mathrm{n}=46)$, and GT $(+)$ : received 9 green tea capsules per day $(\mathrm{n}=37)$. Among the subgroup with a higher provirus load, a decreasing trend in HTLV-1 provirus load values was observed in the $\mathrm{GT}(+)$ group (regression coefficient $(\mathrm{RC})=-0.072, \mathrm{SE}=0.430$ ), but not in the $\mathrm{GT}(-)$ group $(\mathrm{RC}=+0.012, \mathrm{SE}=0.043)$. A significant difference in HTLV-1 provirus load from baseline to each follow-up month between the GT (-) and GT (+) groups observed at the 5 month [12]. 
Summary for human studies: Green tea consumption may preferentially offer some protection against hormone-associated cancer cells, including prostate and breast cancer cells [46]. Although green tea catechins, especially EGCg, strongly inhibit cellular proliferation or cell viability in other cancer cell lines, including oral, esophageal, bladder and skin cells, less information from cohort, case-controlled, or interventional studies on humans have been performed [5]. Recently, Hou et al. reviewed 17 epidemiological studies for stomach cancer, and found that all studies that analyzed men and women separately suggested a reduced risk in women compared to men, albeit the difference was not significant [47]. The inconsistent results between the epidemiologic studies may be due to variables such as differences in tea preparation and consumption, the methods of tea production, the bioavailability of tea compounds, and genetic variation in how the human body responds to tea consumption.

\section{Proposed Mechanism of the Anti-cancer Effects (Experimental Studies)}

We have shown that EGCg markedly inhibits cell proliferation. Furthermore, under identical conditions, EGCg suppressed $B c l-x L$ mRNA expression in non-small-cell lung cancer A549 cells [48]. $\mathrm{Bcl}-\mathrm{xL}$, a member of the Bcl-2 family, inhibits apoptosis by blocking the mitochondrial cytochrome $c$ release $[49,50]$. A decrease in $B c l-x L$ gene expression may lead to the promotion of cell death. We predict that NF- $\mathrm{BB}$ inactivation by catechins, which is an upper stream event, may be essential for the induction of apoptosis. Several cell culture studies have focused on one of the hallmarks of apoptosis induction by green tea catechins, namely NF- $\mathrm{kb}[51,52]$. NF- $\mathrm{\kappa B}$ is a nuclear transcription factor that regulates expression of genes that are critical of the regulation of apoptosis. NF- $\kappa \mathrm{B}$ is bound in a complex in cytosol with inhibitor protein I $\mathrm{K} B$. When I $\mathrm{K} B$ is degraded by proteasomes, NF- $\kappa B$ enters the nucleus and transcript target genes such as apoptosis-inhibiting genes. The suppression of NF- $\kappa \mathrm{B}$ activation may lead to induce apoptosis. Gupta et al. revealed that EGCg reversed the degradation of I $\mathrm{B} a$, inhibitor protein of NF- $\kappa B$, in a cytoplasmic extract. Subsequently $\mathrm{NF}-\kappa \mathrm{B}$ activation is down-regulated and apoptosis is induced in A431 human epidermoid carcinoma cells [53]. On the other hand, the vascular endothelial growth factor (VEGF) is the most critical regulator in development of solid tumors. Indeed, human monoclonal antibody against VEGF, Bevacizumab, is utilized as the molecular target in the clinical use. Recently, Gu et al. reported that EGCG suppresses breast tumor growth by inhibiting the activation of NF- $\kappa B$ and VEGF expression. Oral administration of EGCG significantly reduced tumor VEGF expression in a mouse breast cancer model, in which mammary glands were inoculated with breast cancer cells. It was also shown that EGCG significantly inhibited the activation of NF- $\kappa \mathrm{B}$ in cultured cells [54]. Integrating these evidences, we hypothesized possible mechanism of apoptotic cell death and VEGF suppression by EGCg. That is, EGCg stabilize I $\kappa \mathrm{B}$ and abrogats NF- $\kappa \mathrm{B}$ activation. Then, suppression of the apoptosis-inhibiting protein $\mathrm{Bcl}-\mathrm{xL}$ causes the release of cytochrome $c$ from mitochondria. The activation of caspase signaling induces apoptosis, which is accompanied by a concomitant reduction in VEGF expression might lead to the suppression of angiogenesis (Figure 3).

\section{Green Tea Effect on Lifestyle-related diseases}

Recently, Kokubo et al. reported on the impact of green tea consumption on the incidence of cardiovascular disease and stroke in the Japanese population [55]. In this study, high green tea consumption was found to be inversely associated with the incidence of cardiovascular disease, stroke and stroke subtypes. The result verified by 'the Japan Public Health Center-Based Study Cohort was consistent with distinct study of 'The Tokamachi-Nakasato cohort study in Japan' [56].

Hypertension is one of the major risk factors of cardiovascular disease or stroke. Yang et al. reported that habitual tea consumption of $120 \mathrm{~mL} / \mathrm{d}$ or more for 1 year significantly reduced the risk of developing hypertension in the Chinese population. This demonstrates the negative correlation between the amount and duration of tea consumption on the systolic and diastolic blood pressures. Multiple logistic regression models of the effect of the amount and duration of tea consumption on hypertension have been generated. Compared with non-habitual tea drinkers, the risk of developing hypertension was decreased by $45 \%$ for those who drank 120 to $599 \mathrm{~mL} / \mathrm{d}$, and was further reduced by $65 \%$ for those who drank $600 \mathrm{~mL} / \mathrm{d}$ [6]. One possible mechanism of the effect of green tea on hypertension is the activation of the Akt and endothelial NO synthase (eNOS) pathways. Akt is a serine/threonine protein kinase that is activated by a phosphatidylinositol-3 kinase, which regulates phosphorylation eNOS. eNOS is involved in NO release and vasorelaxation. Ihm et al. assessed the level of phosphorylated Akt and eNOS in aortic rings in a rat model of metabolic syndrome (MS). Although MS rats showed low levels of phosphorylated eNOS and Akt, treatment with a green tea extract (namely, oral administration of 25 $\mathrm{mg} / \mathrm{kg} / \mathrm{d}$ for 12 weeks) increased eNOS and Akt phosphorylation [7].

The effect of green tea administration on serum cholesterol levels is shown. Wu et al. conducted a 2-month controlled green tea intervention study. Postmenopausal women were randomized into three arms: placebo, $400 \mathrm{mg}$ of EGCG as a green tea extract capsule (PPE), or $800 \mathrm{mg}$ of EGCG as a PPE. Total cholesterol and LDL cholesterol decreased significantly in both PPE groups. The LDLcholesterol level differed significantly between the placebo and PPE groups [8]. While the mechanisms by which green tea influences LDL-cholesterol levels remain unknown, animal studies showed that green tea extracts inhibit intestinal absorption of lipids and up regulate lower-density lipoprotein receptors in livermay lead to increase the efflux of cholesterol from liver cells [57].

Most recently, the effect of green tea on blood pressure and lipid profile was assessed by meta-analysis of randomized clinical trial. Based on the results of meta-analysis which included 20 RCTs and 1536 participants, green tea intake results in significant reductions in systolic blood pressure, total cholesterol, and LDL-cholesterol. The

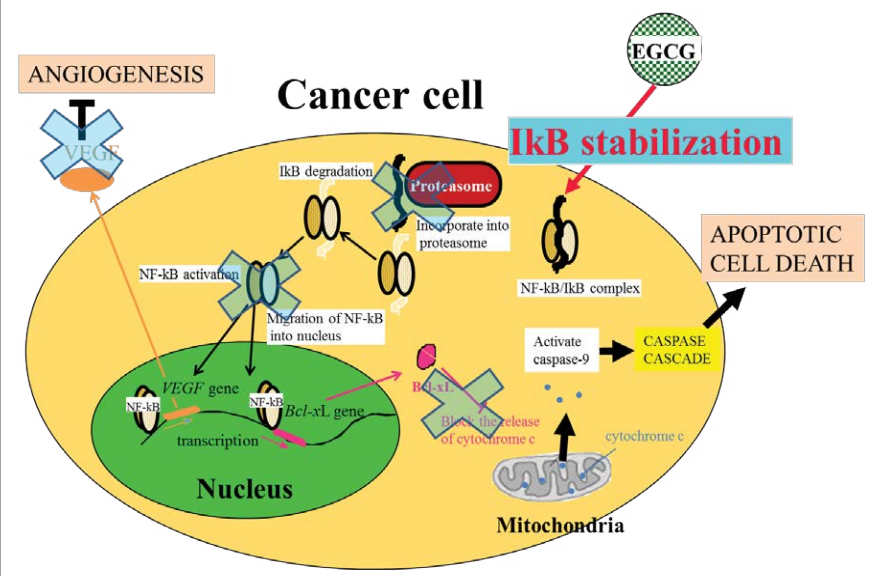

Figure 3: The possible mechanism of apoptotic cell death and antiangiogenesis by EGCG. 
Citation: Sonoda J, Narumi K, Akio K, Erisa T, Toshiro M (2015) Green Tea Catechins -Pharmacokinetic Properties and Health Beneficial Effects. Pharm Anal Acta 6: 333. doi:10.4172/2153-2435.1000333

Page 5 of 6

effects appear greater with longer duration of intervention [58].

\section{Conclusion}

Since green tea catechins absorption after one regular cup of green tea intake is relatively low (estimated in 10 s to $100 \mathrm{~s} \mathrm{nM}$ range), it is difficult to obtain a similar result of anti-cancer effects observed in in vitro cell culture study (in 10 s to $100 \mathrm{~s} \mu \mathrm{M}$ range). However, epidemiological studies shown in this review suggesting that larger portion of green tea consumption for a long term might reduce not only the risk of cancer, but also other lifestyle-related disease incidence. We concluded habitual green tea drinking is good for health.

\section{References}

1. Pastore RL, Fratellone $\mathrm{P}$ (2006) Potential health benefits of green tea (Camellia sinensis): a narrative review. Explore (NY) 2: 531-539.

2. Yang C, Lambert JD, Ju J, Lu G, Sang S (2007) Tea and cancer prevention: molecular mechanisms and human relevance. ToxicolAppIPharmacol 224: 265-273.

3. Du GJ, Zhang Z, Wen XD, Yu C, Calway T, et al. (2012) EpigallocatechinGallate $(E G C G)$ is the most effective cancer chemopreventive polyphenol in greentea. Nutrients 4: 1679-1691.

4. Narumi K, Sonoda J, Shiotani K, Shigeru M, Shibata M, et al. (2014) Simultaneous detection of green tea catechins and gallic acid in human serum after ingestion of green tea tablets using ion-pair high-performance liquid chromatography with electrochemical detection. J Chromatogr B AnalytTechnol Biomed Life Sci 945-946: 147-153.

5. Butt MS, Ahmad RS, Sultan MT, Nasir Qayyum MM, Naz A (2013) Green tea and anticancer perspectives: Updates from last decade. Cirt Rev Food SciNutr 55: 792-805.

6. Yang YC, Lu FH, Wu JS, Wu CH, Chang CJ (2004) The protective effect of habitual tea consumption on hypertension. Arch Intern Med 164: 1534-1540.

7. Ihm SH, Jang SW, Kim OR, Chang K, Oak MH, et al. (2012) Decaffeinated green tea extract improves hypertension and insulin resistance in a rat mode of metabolic syndrome. Atherosclerosis 224: 377-383.

8. Wu AH, Spicer D, Stanczyk FZ, Tseng CC, Yang CS, et al. (2012) Effect of 2-month controlled green tea intervention on lipoprotein cholesterol, glucose and hormone levels in healthy postmenopausal women. Cancer Prev Res 5 393-402.

9. Coimbra S, Santos-Silva A, Rocha-Pereira P, Rocha S, Castro E (2006) Green tea consumption improves plasma lipid profiles in adults. Nutr Res 26: 604-607.

10. Masuda S, Maeda-Yamamoto M, Usui S, Fujisawa T (2014) 'Benifuuki' green tea containing o-methylated catechin reduces symptoms of Japanese cedar pollinosis: a randomized, double-blind, placebo-controlled trial. Allergol Int 63: 211-217

11. Li HC, Yashiki S, Sonoda J, Lou H, Ghosh SK, et al. (2000) Green tea polyphenols induce apoptosis in vitro in peripheral blood T lymphocytes of adult T-cell leukemia patients. Jpn J Cancer Res 91:34-40.

12. Sonoda J, Koriyama C, Yamamoto S, Kozako T, Li HC, et al. (2004) HTLV-1 provirus load in peripheral blood lymphocytes of HTLV-1 carriers is diminished by green tea drinking. Cancer Sci 95: 596-601.

13. Lee MJ, Wang ZY, Li H, Chen L, Sun Y, et al. (1995) Analysis of plasma and urinary tea polyphenols in human subjects. Cancer Epidemiol Biomarkers Prev 4: 393-399

14. Maiani G, Serafini M, Salucci M, Azzini E, Ferro-Luzzi A (1997) Application of a new high-performance liquid chromatographic method for measuring selected polyphenols in human plasma. J Chromatogr B Biomed Sci Appl 692: 311-317.

15. Nakagawa K, Miyazawa T (1997) Chemiluminescence-high-performance liquid chromatographic determination of tea catechin, (-)-epigallocatechin 3-gallate, at picomole levels in rat and human plasma. Anal Biochem 248: 41-49.

16. Lee MJ, Prabhu S, Meng X, Li C, Yang CS (2000) An improved method for the determination of green and black tea polyphenols in biomatrices by highperformance liquid chromatography with coulometric array detection. Anal Biochem 279: 164-169.
17. Arce L, Rios A, Valcarcel M (1998) Determination of anti-carcinogenic polyphenols present in green tea using capillary electrophoresis coupled to a flow injection system. J Chromatogr A 827: 113-120.

18. Roowi S, Stalmach A, Mullen W, Lean ME, Edwards CA, et al. (2010) Green tea flavan-3-ols: colonic degradation and urinary excretion of catabolites by humans. J Agric Food Chem 58: 1296-1304.

19. Umegaki K, Sugisawa A, Yamada K, Higuchi M (2001) Analytical method of measuring tea catechins in human plasma by solid-phase extraction and HPLC with electrochemical detection. J NutrSciVitaminol 47: 402-408.

20. Ishii T, Ichikawa T, Minoda K, Kusaka K, Ito S, et al. (2011) Human serum albumin as an antioxidant in the oxidation of (-)-epigallocatechingallate: participation of reversible covalent binding for interaction and stabilization. BiosciBiotechnolBiochem 75: 100-106.

21. Ishii T, Minoda K, Bae MJ, Mori T, Uekusa Y, et al. (2010) Binding affinity of tea catechins for HSA: characterization by high-performance affinity chromatography with immobilized albumin column. Mol Nutr Food Res 54: 816822.

22. Hong J, Lambert JD, Lee SH, Sinko PJ, Yang CS (2003) Involvement of multidrug resistance-associated proteins in regulating cellular levels of (-)-epigallocatechin-3-gallate and its methyl metabolites. BiochemBiophys Res Commun 310: 222-227.

23. Zhang L, Zheng Y, Chow MS, Zuo Z (2004) Investigation of intestinal absorption and disposition of greenteacatechins by Caco-2 monolayer model. Int J Pharm 287: $1-12$

24. Song Q, Li D, Zhou Y, Yang J, Yang W, et al. (2014) Enhanced uptake and transport of (+)-catechin and (-)-epigallocatechingallate in niosomal formulation by human intestinal Caco-2 cells. Int J Nanomedicine 9: 2157-2165.

25. Hung CC, Chiou MH, Teng YN, Hsieh YW, Huang CL, et al. (2013) Functional impact of $A B C B 1$ variants on interactions between P-glycoprotein and methadone. PloS One 8: e59419.

26. Hoffmeyer S, Burk O, von Richter O, Arnold HP, Brockmöller J, et al. (2000) Functional polymorphisms of the human multidrug-resistance gene: multiple sequence variations and correlation of one allele with $\mathrm{P}$-glycoprotein expression and activity in vivo. ProcNatlAcadSci U S A 97: 3473-3478.

27. Misaka S, Yatabe J, Müller F, Takano K, Kawabe K, et al. (2014) Green tea ingestion greatly reduces plasma concentrations of nadolol in healthy subjects. ClinPharmacolTher 95: 432-438.

28. Hong J, Lu H, Meng X, Ryu JH, Hara Y, et al. (2002) Stability, cellular uptake, biotransformation, and efflux of tea polyphenol (-)-epigallocatechin-3-gallate in HT-29 human colon adenocarcinoma cells.Cancer Res 62: 7241-7246.

29. Imai K, Suga K, Nakachi K (1997) Cancer-preventive effects of drinking green tea among a Japanese population. Prev Med 26: 769-775.

30. Kurahashi N, Sasazuki S, Iwasaki M, Inoue M, Tsugane S (2007) Green tea consumption and prostate cancer risk in Japanese men: a prospective study. Am J Epidemiol 167: 71-77.

31. Bettuzzi S, Brausi M, Rizzi F, Castagnetti G, Peracchia G, et al. (2006) Chemoprevention of human prostate cancer by oral administration of green tea catechins in volunteers with high-grade prostate intraepithelial neoplasia: a preliminary report from a one-year proof-of-principle study. Cancer Res 66 : 1234-1240.

32. Zhang M, Holman CD, Huang JP, Xie X (2007) Green tea and the prevention of breast cancer: a case-control study in Southeast China. Carcinogenesis 28 : 1074-1078.

33. Suzuki Y, Tsubono Y, Nakaya N, Suzuki Y, Koizumi Y, et al. (2004) Green tea and the risk of breast cancer: pooled analysis of two prospective studies in Japan. Br J Cancer 90: 1361-1363.

34. Li MJ, Yin YC, Wang J, Jiang YF (2014) Green tea compounds in breast cance prevention and treatment. World J ClinOncol 5: 520-528.

35. Ogunleye AA, Xue F, Michels KB (2010) Green tea consumption and breas cancer risk or recurrence: a meta-analysis. Breast Cancer Res Treat 119: 477 484.

36. Nakachi K, Suemasu K, Suga K, Takeo T, Imai K, et al. (1998) Influence of drinking green tea on breast cancer malignancy among Japanese patients. Jpn J Cancer Res 89: 254-261. 
Citation: Sonoda J, Narumi K, Akio K, Erisa T, Toshiro M (2015) Green Tea Catechins -Pharmacokinetic Properties and Health Beneficial Effects. Pharm Anal Acta 6: 333. doi:10.4172/2153-2435.1000333

37. Inoue M, Tajima K, Mizutani M, Iwata H, Iwase T, et al. (2001) Regular consumption of green tea and the risk of breast cancer recurrence: followup study from the Hospital-based Epidemiologic Research Program at Aich Cancer Center (HERPACC), Japan. Cancer Lett167: 175-182.

38. Key TJ, Sharp GB, Appleby PN, Beral V, Goodman MT, et al. (1999) Soya foods and breast cancer risk: a prospective study in Hiroshima and Nagasaki, Japan. Br J Cancer 81: 1248-1256.

39. Tao M, Liu D, Gao L, Jin F (2002) Association between green tea drinking and breast cancer risk. Tumor 22: 11-15.

40. Inoue M, Robien K, Wang R, Van Den Berg DJ, Koh WP, et al. (2008) Green tea intake, MTHFR/TYMS genotype and breast cancer risk: the Singapore Chinese Health Study. Carcinogenesis 29: 1967-1972.

41. Shrubsole MJ, Lu W, Chen Z, Shu XO, Zheng Y, et al. (2009) Drinking green tea modestly reduces breast cancer risk. J Nutr 139: 310-316.

42. Yuan JM, Gao YT, Yang CS, Yu MC (2007) Urinary biomarkers of tea polyphenols and risk of colorectal cancer in the Shanghai Cohort Study. Int J Cancer 120: 1344-1350.

43. Ohno Y, Wakai K, Genka K, Ohmine K, Kawamura T, et al. (1995) Tea consumption and lung cancer risk: a case-control study in Okinawa, Japan. Jpn J Cancer Res 86: 1027-1034

44. Li Q, Kuriyama S, Kakizaki M, Yan H, Sone T, et al. (2010) History of diabetes mellitus and the risk of prostate cancer: the Ohsaki Cohort Study. Cance Causes Control 21: 1025-1032.

45. Wang L, Zhang X, Liu J, Shen L, Li Z (2014) Tea consumption and lung cance risk: a meta-analysis of case-control and cohort studies. Nutrition 30: 1122-1127.

46. Stuart EC, Scandlyn MJ, Rosengren RJ (2006) Role of epigallocatechingallate (EGCG) in the treatment of breast and prostate cancer. Life Sci 79: 2329-2336.

47. Hou IC, Amarnani S, Chong MT, Bishayee A (2013) Green tea and the risk of gastric cancer: epidemiological evidence. World J Gastroenterol. 19: 3713-3722.

48. Sonoda JI, Ikeda R, Baba Y, Narumi K, Kawachi A, et al. (2014) Green tea catechin, epigallocatechin-3-gallate, attenuates the cell viability of human nonsmall-cell lung cancer A549 cells via reducing Bcl-xL expression. ExpTher Med 8: $59-63$
49. Lee JH, Jeong YJ, Lee SW, Kim D, Oh SJ, et al. (2010) EGCG induces apoptosis in human laryngeal epidermoid carcinoma Hep2 cells via mitochondria with the release of apoptosis-inducing factor and endonuclease G. Cancer Lett 290: 68-75

50. Roy P, Nigam N, George J, Srivastava S, Shukla Y (2009) Induction of apoptosis by tea polyphenols mediated through mitochondrial cell death pathway in mouse skin tumors. Cancer Biol Ther 8: 1281-1287.

51. Hastak K, Gupta S, Ahmad N, Agarwal MK, Agarwal ML, et al. (2003) Role of p53 and NF-kappaB in epigallocatechin-3-gallate-induced apoptosis of LNCaP cells. Oncogene 22:4851-4859.

52. Han DW, Lee MH, Kim HH, Hyon SH, Park JC (2011) Epigallocatechin-3gallate regulates cell growth, cell cycle and phosphorylated nuclear factor-kB in human dermal fibroblasts. Acta Pharmacol Sin 32:637-46.

53. Gupta S, Hastak K, Afaq F, Ahmad N, Mukhtar H (2004) Essential role of caspases in epigallocatechin-3-gallate-mediated inhibition of nuclear factor kappa B and induction of apoptosis. Oncogene 23: 2507-2522.

54. Gu JW, Makey KL, Tucker KB, Chinchar E, Mao X, et al. (2013) EGCG, major green tea catechin suppresses breast tumor angiogenesis and growth via inhibiting the activation of HIF-1 $\alpha$ and NFKB, and VEGF expression. Vasc Cell 5: 9.

55. Kokubo Y, Iso H, Saito I, Yamagishi K, Yatsuya H, et al. (2013) The impact of green tea and coffee consumption on the reduced risk of stroke incidence in Japanese population: the Japan public health center-based study cohort. Stroke 44: 1369-1374

56. Tanabe N, Suzuki H, Aizawa Y, Seki N (2008) Consumption of green and roasted teas and the risk of stroke incidence: results from the TokamachiNakasato cohort study in Japan. Int J Epidemiol 37: 1030-1040.

57. Bursill CA, Roach PD (2007) A green tea catechin extract up regulates the hepatic low-density lipoprotein receptor in rats. Lipids 42:621-627.

58. Onakpoya I, Spencer E, Heneghan C, Thompson M (2014) The effect of greentea on blood pressure and lipid profile: a systematic review and metaanalysis of randomized clinical trials. NutrMetabCardiovasc Dis 24: 823-836. 